\title{
Correction to: mRNA and microRNA profiles are associated with stress susceptibility and resilience induced by psychological stress in the prefrontal cortex
}

\author{
Jiuyong Yang ${ }^{1}$ • Jinyan Sun ${ }^{1}$ - Yanjun $\mathrm{Lu}^{1} \cdot{\text { Tingting } A n^{1} \cdot \text { Wei Lu }}^{1} \cdot$ Jin-Hui Wang ${ }^{1,2,3}$ \\ Published online: 24 July 2020 \\ (C) Springer-Verlag GmbH Germany, part of Springer Nature 2020
}

\section{Correction to: Psychopharmacology}

https://doi.org/10.1007/s00213-020-05593-x

The title of this article is "mRNA and microRNA profiles are associated with stress susceptibility and resilience induced by psychological stress in the prefrontal cortex". However, the title was mistakenly summed as "Revision to psychopharmacology mRNA and microRNA profiles are associated with stress susceptibility and resilience induced by psychological stress in the prefrontal cortex "(as seeing in https://doi.org/10. 1007/s00213-020-05593-x) at the proof stage. The words "Revision to Psychopharmacology" was just a revision mark, not part of the article's title.

We are very sorry that we did not find this mistake in the proof process.

Publisher's note Springer Nature remains neutral with regard to jurisdictional claims in published maps and institutional affiliations.

The online version of the original article can be found at https://oi.org/ $10.1007 / \mathrm{s} 00213-020-05593-\mathrm{x}$

Wei Lu

luwei@qdu.edu.cn

$\triangle$ Jin-Hui Wang

jhw@ibp.ac.cn

1 School of Pharmacy, Qingdao University, 38 Dengzhou, Qingdao 266021, Shandong, China

2 University of Chinese Academy of Sciences, Beijing 100049, China

3 Institute of Biophysics, University of Chinese Academy of Sciences, 15 Datun Road, Chaoyang District, Beijing 100101, China 